\title{
Diagnosis of Brain Diseases using Neural Networks
}

\author{
Anagha Naga Krishna, Tejashwini V, Sudhamani M J
}

\begin{abstract}
Intensification in the occurrence of brain diseases and the need for the initial diagnosis for ailments like Tumor, Alzheimer's, Epilepsy and Parkinson's has riveted the attention of researchers. Machine learning practices, specifically deep learning, is considered as a beneficial diagnostic tool. Deep learning approaches to neuroimaging will assist computer-aided analysis of neurological diseases. Feature extraction of neuroimages carried out using Artificial Neural Networks leads to better diagnoses. In this study, all the brain diseases are revisited to consolidate the methodologies carried out by various authors in the literature.
\end{abstract}

Keywords: Brain, Classification, Feature Extraction, Neural network

\section{INTRODUCTION}

An artificial neural network (ANN) is a simulated imitation of the functionality of the neurons present in the human brain. It is a computational representation that is based on the structure and functionality of the parallel nature of the brain. The human body can best exemplify the property of parallel processing. Inspired by the biological nervous system, an ANN forms an interconnection of processing elements (nodes) that can operate parallelly. A simple architecture is shown in Fig.1. Along with the interconnection, the processing elements are also layered. A small set of contiguous processing elements form a layer. Most artificial neural networks compulsorily consist of an input and output layer, along with some supplementary layers called hidden layers. The neural interconnection is trained to obtain the desired output by performing a certain elements(weights) can be altered to make the network perform the desired function. The interconnection of neurons leads to the formation of multiple pathways to reach the desired output and hence the connections determine the working and functionality of the network [1]. The data that flows through the network will affect the configuration of the ANN, indicating that the network has learned based on the input and output.

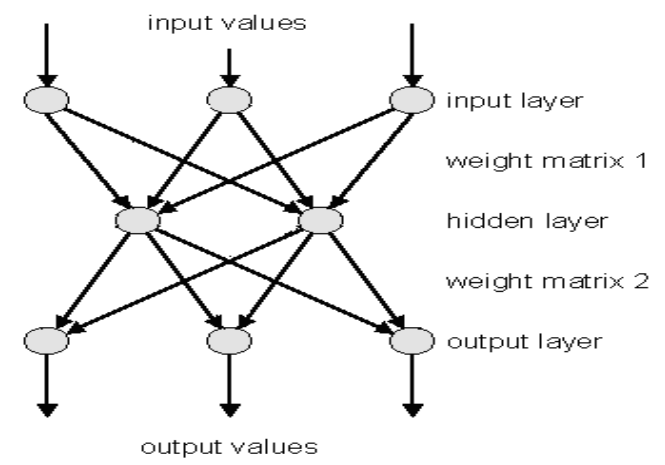

Fig. 1. A simple neural network

Revised Manuscript Received on December 15, 2019.

Ms. Anagha Naga Krishna, B.E. in Computer Science and Engineering at RNSIT, Bengaluru.

Dr. M J Sudhamani, Assistant Professor in Dept. of Computer Science and Engineering at RNSIT, Bengaluru.

Tejashwini V, Assistant Professor in Dept. of Computer Science and Engineering at RNSIT, Bengaluru. function. The values of the connections between the

The implementation of ANN is found In the field of medical diagnosis because a neural network can learn by examples and the process of identifying diseases need not be provided. They can be efficiently trained using labeled examples and adjusting the connection weights [1]. ANNs must be supplied with a collection of examples that contain all the variations of the diseases. Also, ANNs are able to tolerate a decent level of noise in the data and consequently they provide sufficient accuracy in the classification or prediction.

\section{DIAGNOSIS OF BRAIN DISEASES}

\section{a. Detection of brain tumor}

A brain tumor is a very hazardous and precarious disease because the affected cells can easily split and spread to other regions. Hence, it must be detected as early as possible to avoid

further consequences. The conventional method for detecting the tumor diseases using human magnetic resonance imaging (MRI) brain images is done by physicians manually, but this technique has a very high possibility of causing human errors and may not be very accurate. Computerized classification of tumors using MRI images must be highly accurate and must have no potential of causing errors. With the help of artificial neural networks, the brain images obtained through the process of MRI can be classified to identify the type of tumor [2].

Gonge et al [2] proposed a study that involved the processing and classification of the MRI brain images. The extraction of texture features was done using a Gray Level Co-occurrence Matrix (GCLM). The image processing consisted of a three-stage process. First was the segmentation, Segmentation divides an image into smaller components, and continues till the border of the tumor is reached.

Next was the feature extraction which was performed using a Gray Level Co-occurrence Matrix. Later an ANN classifier was designed to distinguish between normal and abnormal brain images.

Abdalla et al [3] used the process of texture analysis which used The Spatial Gray Level Dependency matrix (SGLD) matrix generator, which decomposed the input image into texture features (Haralick's features). A backpropagation network with supervised learning was used, which is the best method for samples having a nonlinear transformation like sigmoid transfer function. Fourteen parameters were used to calculate the Haralick's features for each brain image, out of which eight parameters were chosen in random each time and a graph was plotted. Finally, the best eight parameters were formulated, and the devised algorithm provided an accuracy of $99 \%$, and sensitivity of $97.9 \%$.

Al-Badarneh et al [4] worked with the brain Images from MRI and performed the classification to determine whether the brain images are normal or abnormal. 


\section{Diagnosis of Brain Diseases using Neural Networks}

The authors performed a feature extraction. Out of 65,536 features, 19 were selected. The authors then used two major methods to classify the images. The extracted features of the MRI were fed to the ANN and a K-nearest neighbors (K-NN) classifier. On observing the experimental results, it was concluded that the K-NN classifier provided an accuracy of $100 \%$ and the ANN provided an accuracy of $98.92 \%$.

Amsaveni et al [5] had used performed the feature extraction by Gabor Filter. The author used histogram equalization in the step of preprocessing which is for obtaining better accuracy. Gabor representation has been shown to be ideal for minimizing the two-dimensional uncertainty in space and frequency. Gabor filters have been used in several image classification and segmentation applications. The Gabor function along with the Fourier transform lead to the formation of Gabor wavelets, and wavelet transform. From which a feature vector is formed. In this study, a backpropagation algorithm with the feedforward technique was used for the classification. However, the method of wavelet transform has proven to not give a very high accuracy. The experiment showed an accuracy of $89.9 \%$.

Sharif et al [6] had used the multilayer feedforward neural network along with backpropagation training algorithm on Positron Emission Tomography (Pet) images for tumor detection. The authors in the initial stage subjected the image to histogram equalization and median filter to intensify the standard of image features and also to eliminate noise. This image was further processed by applying Thresholding and Magnetic resonance angiogram (MRA) approaches and their results were fed to ANN1 and ANN2 respectively. In the final stage, the results of both the ANNs were compared and the best result was considered. It was observed that the outputs of ANN2 were more reliable compared to the outputs of ANN1.

Murugesan et al [7] had projected a system for automated diagnosis of a brain tumor in Electroencephalogram (EEG) signals using a feedforward backpropagation neural network. The artifacts existing in the signal were eliminated by applying adaptive filtering. Later on, by applying fast Fourier transform (FFT) the generic features were obtained. The clean EEG signal was given as input to the ANN for training and testing. Out of 325 samples of EEG which consisted of both normal and abnormal signals the detection accuracy of the system for normal was $94.4785 \%$ and for abnormal was $98.7654 \%$.

Azadeh et al [8] had used multilayer perceptron neural networks for the classification of brain tumors from Magnetic Resonance Spectroscopic Imaging (MSRI) data. The authors initially used wavelets and wavelet packets to reduce the noise in the signal and the baseline distortions were also removed. The features were obtained by applying two different approaches, wavelets and wavelet packets and were fed to ANN to identify the abnormal spectra and abnormality type. It was found that brain lesions impacted the entire signal along with metabolite peaks. This system showed a classification accuracy of $72 \%$ for clinical data and $83 \%$ for simulated data.

Krishnammal et al [9] suggested a system for identifying abnormalities in the brain using the Convolution Neural Network (CNN). The MRI images of the brain were taken from public databases and were preprocessed. The feature extraction was done by curvelet transform and GLCM and fed to CNN. The authors used AlexNet CNN model for image classification and K-Means Algorithm for segmentation that is it evaluates tumor detection. $100 \%$ accuracy was achieved by $\mathrm{CNN}$ during training and validation phase, but more time will be consumed for training CNN.

\section{b. Detection of Alzheimer's disease}

Alzheimer's disease (AD) is a cumulative mental deterioration that damages memory and other prime mental functions. It is also the most common cause of dementia. $\mathrm{AD}$ causes the death of nerve cells and loss of tissue throughout the brain, therefore decreasing the brain volume in size dramatically through time and affecting most of its functions [10].

Authors in [10] used a three-dimensional adaptive $\mathrm{CNN}$ for the diagnosis of Alzheimer's disease. Out of the three methods of structural MRI (sMRI), functional MRI (fMRI), and positron emission tomography (PET), The sMRI has been recognized as a promising indicator of the AD progression. The proposed model used a 3D convolutional autoencoder to perform the feature extraction of the brain images of MRI. Next was the classification of AD by using adaptive $3 \mathrm{D}$ CNN. Since $\mathrm{CNN}$ was used, the lower layers also had to be pre-trained and fine-tuned. This experiment used the reLU activation function at each inner layer and the completely connected upper layers predicted whether the input was normal or abnormal. The output was able to distinguish between a normal case, a case with mild cognitive impairment and an affected case.

Work proposed in [11] a method to detect Alzheimer's disease using the siamese convolutional neural network (CNN). Convolutional neural network belongs to the section of deep neural networks, most commonly applied to analyzing visual imagery. $\mathrm{CNN}^{\prime} \mathrm{s}$ use relatively little preprocessing compared to other image classification algorithms. The Siamese CNN network contains three CNN branches, which each branch is for one type of image. The images were randomly selected and sent to ResNet, where a pairwise distance was formed with which a triple loss function was formulated. An extracting feature vector was formed, called as embedding vector for a Siamese CNN.

After training with the triple loss function, the classifier provided an accuracy of $98.72 \%$.

Proposal in [12] worked with incremental learning on a multi-layer neural network to aid the diagnosis of Alzheimer's disease. In this study, the ANN training was performed with Cerebral Blood Flow (CBF) measurements from Single Photon Emission Computed Tomography (SPECT). The study also used incremental learning which enables the learning to start where it left off and not go back to the initial state. First, the ANN model was trained using Unsupervised learning and then an offset algorithm was used along with incremental learning which used supervised learning and dynamically built layered networks respectively. The experiment was performed on a small data set and the results were not comparable.

Patil et al [13] had projected the use of feed-forward neural networks for automated multi-class detection of Dementia from MRI images. In this method, the 1D histogram signal was attained from 2D MR brain images and Discrete Cosine Transform was used to compress it further. 
For each image six 6 absolute largest coefficients of Discrete Cosine Transform were considered and were utilized to train the four-class classifier model. The rate of classification for this system was $100 \%$ for a collection of MR brain images from the whole brain atlas database.

\section{c. Diagnosis of Epilepsy}

Epilepsy is a neurological dysfunction in which nerve cell activity in the brain is deranged, causing seizures. Epilepsy can cause sudden movements or jerks in the arms or legs. Some common diagnostic methods for epilepsy are magnetic resonance (MR), brain tomography (BT). But electroencephalogram (EEG) is commonly used for studying the epileptic seizures, in neurological centers.

Akin et al in his study [14] worked on wavelet transform and ANN to classify the EEG signals to identify epilepsy. The EEG signal was separated into four spectral components, the EEG signal is a non-stationary signal, hence the frequency components were extracted using a short-time Fourier transform and wavelet transform. The Wavelet Transform decomposes a signal onto a set of functions called wavelets. The wavelet transform is separated into continuous and discrete for providing the input signal. Depending on the EEG signal components, an artificial neural network was developed. The EEG signal components were given as input to the neural network. The ANN classifier gave a distinction between epileptic case, normal case and pathologic case. The accuracy provided by the neural network turned out to be $97 \%$ for epileptic case, $98 \%$ for healthy case, and $93 \%$ for a pathologic case with an average of $96 \%$. However, wavelet transform requires a lot of storage and computational cost is high.

Khalil et al [15] proposed a technique which made use of Discrete wavelet transform (DWT) for splitting the EEG signal. The signal comprised of various frequency sub-band which are: alpha, beta, gamma, delta and theta. On the application of DWT, many segments of detailed coefficients were formed, which was the result of feature extraction. Shannon entropy was used on the coefficients for minimizing the measured dimensions of the extracted features. The resultant was then fed into a feed forward neural network classifier. The classifier was used to effectively distinguish between normal case and epileptic case for the appropriate EEG signal. This approach provided efficient results and the accuracy was $100 \%$.

\section{d. Detection of Parkinson's Disease}

Parkinson's disease is a degenerative ailment of the central nervous system identified by impaired muscular coordination and tremor. It is usually caused by the deterioration of neurons in the substantia nigra. This leads to a lack of dopamine, which is crucial for balanced and smooth muscle movements in our body. Therefore, the absence of which results in abnormal nerve functioning.

A work proposed in [16] put forward a prediction model to diagnose Parkinson's Disease at an early stage with the help of image processing and ANN. In this model, the Authors had used the Single-photon Emission Tomography (SPECT) images that were collected from PPMI database. These images were preprocessed by using spatial normalization, binary mask, and unsharp masking before detecting the Region of Interest that is the right and left putamen and caudate. Based on this, the area of ROI was measured and was given as input to ANN for training it. This model had an accuracy of $94 \%$, a specificity of $88 \%$ and a sensitivity of $100 \%$.

Gharehchopogh et al [17] used A convolutional three-layer MLP network with 5 hidden neurons and RBF with 136 hidden neurons to diagnosis Parkinson's disease. The database used was taken from the UCI repository. Out of 59 instances, the MLP could classify 55 correctly whereas RBF could classify only 51 correctly.

\section{RESULTS AND DISCUSSIONS}

Table 1.1 consolidates all the methodologies used for brain turmor detection along with results.

Table 1.1 List of highlighting all brain diseases, methodologies along with the result

\begin{tabular}{|l|l|l|l|}
\hline \multicolumn{4}{|l|}{ Detection of brain tumor } \\
\hline Authors & Year & Methodology & Result \\
\hline $\begin{array}{l}\text { Rajeshwar Nalbalwar,Umakant } \\
\text { Majhi, Raj Patil, } \\
\text { Prof.Sudhanshu Gonge [2] }\end{array}$ & 2014 & $\begin{array}{l}\text { Feature extraction is performed using } \\
\text { GCLM. ANN is used for } \\
\text { classification. }\end{array}$ & $\begin{array}{l}\text { The proposed approach } \\
\text { provides an appreciable } \\
\text { efficiency with an improved } \\
\text { sensitivity and accuracy }\end{array}$ \\
\hline $\begin{array}{l}\text { Hussna Elnoor Mohammed } \\
\text { Abdalla, M. Y. Esmail [3] }\end{array}$ & 2018 & $\begin{array}{l}\text { Feature extraction of MRI brain } \\
\text { images is performed by using } \\
\text { Haralick's features with the } \\
\text { foundation of SGLD, which is } \\
\text { classified by using a neural network } \\
\text { classifier. }\end{array}$ & $\begin{array}{l}\text { The trained network } \\
\text { successfully provides an } \\
\text { accuracy of 99\%, and a } \\
\text { sensitivity of 97.9\%. }\end{array}$ \\
\hline
\end{tabular}


Diagnosis of Brain Diseases using Neural Networks

\begin{tabular}{|c|c|c|c|}
\hline $\begin{array}{l}\text { Amer Al-Badarneh, Hassan } \\
\text { Najadat, Ali M. Alraziqi [4] }\end{array}$ & 2012 & $\begin{array}{l}\text { Extraction of texture features and } \\
\text { classification of MRI images through } \\
\text { K-NN and ANN }\end{array}$ & $\begin{array}{l}\text { The accuracy provided by } \mathrm{K}- \\
\mathrm{NN} \text { is } 100 \% \text { and ANN is } \\
98.92 \%\end{array}$ \\
\hline V. Amsaveni [5] & 2013 & $\begin{array}{l}\text { Gabor filter and wavelet transform } \\
\text { used for feature extraction and } \\
\text { backpropagation network as a } \\
\text { classifier }\end{array}$ & $\begin{array}{l}\text { The classification provides } \\
\text { an accuracy of } 89.9 \% \text {. }\end{array}$ \\
\hline $\begin{array}{l}\text { Mhd Saeed Sharif, Abbes Amira } \\
\text { [6] }\end{array}$ & 2009 & $\begin{array}{l}\text { Multilayer ANN, multiresolution } \\
\text { analysis, and } \\
\text { Thresholding is used on PET images. }\end{array}$ & $\begin{array}{l}\text { This system was } \\
\text { considerably good at tumor } \\
\text { quantification and detection. }\end{array}$ \\
\hline $\begin{array}{l}\text { M. Murugesan,Dr. (Mrs.) } \\
\text { R.Sukanesh [7] }\end{array}$ & 2009 & $\begin{array}{l}\text { Adaptive filtering and FFT are used } \\
\text { to produce a clean EEG signal and is } \\
\text { fed to a feedforward backpropagation } \\
\text { neural network. }\end{array}$ & $\begin{array}{l}\text { Detection accuracy of } \\
94.4785 \% \text { was achieved for } \\
\text { normal and an accuracy of } \\
98.7654 \% \text { was achieved for } \\
\text { abnormal. }\end{array}$ \\
\hline $\begin{array}{l}\text { Azadeh Yazdan-Shahmorad, } \\
\text { Hamid Soltanian-Zadeh, Reza } \\
\text { A. Zoroofi [8] }\end{array}$ & 2004 & $\begin{array}{l}\text { Wavelets and wavelet packets are } \\
\text { utilized to obtain the features from } \\
\text { MRSI data. } \\
\text { ANN is used to find the abnormal } \\
\text { spectra and abnormality type. }\end{array}$ & $\begin{array}{l}\text { The system showed a } \\
\text { classification accuracy of } \\
72 \% \text { for clinical data and } \\
83 \% \text { for simulated data. }\end{array}$ \\
\hline $\begin{array}{l}\text { P. Muthu Krishnammal and } \\
\text { S.Selvakumar Raja [9] }\end{array}$ & 2019 & $\begin{array}{l}\text { Feature extraction from MRI images } \\
\text { is done by FDCT and GLCM. CNN } \\
\text { is used for classification and K- } \\
\text { Means for image segmentation }\end{array}$ & $\begin{array}{l}100 \% \text { accuracy was achieved } \\
\text { by } \mathrm{CNN} \text { during the training } \\
\text { and validation phase but } \\
\text { more time is consumed for } \\
\text { training CNN. }\end{array}$ \\
\hline \multicolumn{4}{|c|}{ Detection of Alzheimer's disease } \\
\hline Authors & Year & Methodology & Result \\
\hline $\begin{array}{l}\text { Ehsan Hosseini-Asl, Robert } \\
\text { Keynton, Ayman El-Baz [10] }\end{array}$ & 2016 & $\begin{array}{l}\text { Variations in the MRI scans are } \\
\text { examined by a } 3 \mathrm{D} \text { CNN and an } \\
\text { autoencoder }\end{array}$ & $\begin{array}{l}\text { The experiment provides an } \\
\text { average accuracy of } 92.56 \% \text {. }\end{array}$ \\
\hline $\begin{array}{l}\text { Mostafa Amin-Naji, Hami } \\
\text { Mahdavinataj, Ali } \\
\text { Aghagolzadeh [11] }\end{array}$ & 2014 & $\begin{array}{l}\text { Uses siamese CNN on the data } \\
\text { obtained by structural MRI }\end{array}$ & $\begin{array}{l}\text { Accuracy of } 98.72 \% \text { is } \\
\text { obtained from this approach }\end{array}$ \\
\hline $\begin{array}{l}\text { Marie Chan, Bernard Andre, } \\
\text { Armando Herrera, Pierre Celsis } \\
{[12]}\end{array}$ & 1993 & $\begin{array}{l}\text { Unsupervised and incremental } \\
\text { learning is used. The network is } \\
\text { trained with the aid of CBF from } \\
\text { SPECT }\end{array}$ & $\begin{array}{l}\text { The proposed approach was } \\
\text { tested on a small data set, } \\
\text { output was not comparable }\end{array}$ \\
\hline $\begin{array}{l}\text { Prof.Mrs.M.M.Pati, } \\
\text { Prof.A.R.Yardi [13] }\end{array}$ & 2013 & $\begin{array}{l}\text { Feed-forward neural networks were } \\
\text { used for automated multi-class } \\
\text { detection of Dementia from MRI } \\
\text { images which were compressed by } \\
\text { using DCT. }\end{array}$ & $\begin{array}{l}\text { A classification rate of } 100 \% \\
\text { was achieved from the } \\
\text { proposed system. }\end{array}$ \\
\hline
\end{tabular}




\section{Diagnosis of Epilepsy}

\begin{tabular}{|l|l|l|l|}
\hline Authors & Year & Methodology & Result \\
\hline $\begin{array}{l}\text { M.Akin, M.A.Arserim, } \\
\text { M.K.Kiymik, I.Turkoglu [14] }\end{array}$ & 2001 & $\begin{array}{l}\text { Used wavelet transform to disconnect } \\
\text { the spectral components of EEG } \\
\text { signals }\end{array}$ & $\begin{array}{l}\text { The accuracy provided by } \\
\text { the neural network is an } \\
\text { average of 96\% }\end{array}$ \\
\hline $\begin{array}{l}\text { Khalil AlSharabi, Sutrisno } \\
\text { Ibrahim, Ridha Djemal, } \\
\text { Abdullah Alsuwailem [15] }\end{array}$ & 2016 & $\begin{array}{l}\text { Used DWT, Shannon } \\
\text { entropy and FFNN }\end{array}$ & $\begin{array}{l}\text { Provided an accuracy of } \\
100 \%\end{array}$ \\
\hline
\end{tabular}

Detection of Parkinson's Disease

\begin{tabular}{|c|c|c|c|}
\hline Authors & Year & Methodology & Result \\
\hline $\begin{array}{l}\text { Mosarrat Rumman1, Abu } \\
\text { Nayeem Tasneem1, Sadia } \\
\text { Farzana1, Monirul Islam Pavel1 } \\
\text { and Dr. Md. Ashraful Alam1 } \\
\text { [16] }\end{array}$ & 2018 & $\begin{array}{l}\text { Adaptive thresholding and sequential } \\
\text { grass-fire algorithm were used for } \\
\text { ROI detection and area calculation } \\
\text { from the preprocessed SPECT } \\
\text { images and were fed to ANN. }\end{array}$ & $\begin{array}{l}\text { The accuracy achieved by } \\
\text { the proposed system is } 94 \% \text {. }\end{array}$ \\
\hline $\begin{array}{l}\text { Farhad Soleimanian } \\
\text { Gharehchopogh, Peyman } \\
\text { Mohammadi. [17] }\end{array}$ & 2013 & $\begin{array}{l}\text { MLP and RDF ANNs were utilized } \\
\text { to distinguish between those who } \\
\text { were suffering and who were not. }\end{array}$ & $\begin{array}{l}\text { A classification accuracy of } \\
93.22 \% \text { was achieved by } \\
\text { MLP where as an accuracy } \\
\text { of } 86.44 \% \text { was achieved by } \\
\text { RBF. }\end{array}$ \\
\hline
\end{tabular}

\section{CONCLUSION}

This study discusses all the different techniques in which artificial neural networks can be used for medical diagnosis in the field of neurology. The brain images undergo a feature extraction, after which the images are classified with a neural network. Out of the various methods used for feature extraction, the right selection plays a significant role in the correctness and accuracy of the classification. For the detection of brain tumor, the method using a K-NN classifier [4] provided an accuracy of $100 \%$. Detection of Alzheimer's also achieved a classification rate of $100 \%$ by using a feed forward neural network and DCT [13]. The usage of DWT, Shannon entropy and FFNN [15] provided an accuracy of $100 \%$ for the diagnosis of Epilepsy. An accuracy of $94 \%$ was obtained for the detection of Parkinson's disease using SPECT images and sequential grass-fire algorithm [16].

\section{REFERENCES}

1. Qeethara Kadhim Al-Shayea, "Artificial Neural Networks in Medical Diagnosis", IJCSI International Journal of Computer Science Issues, Vol. 8, Issue 2, March 2011.

2. Rajeshwar Nalbalwar,Umakant Majhi ,Raj Patil,Prof.Sudhanshu Gonge, "Detection of Brain Tumor by using ANN", International Journal of Research in Advent Technology, Vol.2, No.4, April 2014.

3. Hussna Elnoor Mohammed Abdalla, M. Y. Esmail, "Brain Tumor Detection by using Artificial Neural Network", 2018 International Conference on Computer, Control, Electrical, and Electronics Engineering (ICCCEEE).

4. Amer Al-Badarneh, Hassan Najadat, Ali M. Alraziqi, "A Classifier to Detect Tumor Disease in MRI Brain Images", 2012 IEEE/ACM International Conference on Advances in Social Networks Analysis and Mining.

5. V. Amsaveni, "Detection of Brain Tumor using Neural Network", 4th ICCCNT - 2013, July 4-6, 2013, Tiruchengode, India.

6. Mhd Saeed Sharif, Abbes Amira, "AN INTELLIGENT SYSTEM FOR PET TUMOUR DETECTION AND QUANTIFICATION”, 978-14244-5654-3/09/\$26.00 (C2009 IEEE

7. M. Murugesan, Dr. (Mrs) R.Sukanesh, "Automated Detection of Brain Tumor in EEG Signals Using Artificial Neural Networks”, 2009 International Conference on Advances in Computing, Control, and Telecommunication Technologies

8. Azadeh Yazdan-Shahmorad, Hamid Soltanian-Zadeh, Reza A. Zoroofi, "MRSI Brain Tumor Characterization Using Wavelet and Wavelet Packets Feature Spaces and Artificial Neural Networks", Proceedings of the $26^{\text {th }}$ Annual International Conference of the IEEE EMBS San Francisco, CA, USA, September 1-5,2004

9. P. Muthu Krishnammal, S.Selvakumar Raja, "Convolutional Neural Network based Image Classification and Detection of Abnormalities in MRI Brain Images", International Conference on Communication and Signal Processing, April 4-6, 2019,India.

10. Ehsan Hosseini-Asl, Robert Keynton, Ayman El-Baz, "ALZHEIMER'S DISEASE DIAGNOSTICS BY ADAPTATION OF 3D CONVOLUTIONAL NETWORK”, ICIP 2016.

11. Mostafa Amin-Naji, Hami Mahdavinataj, Ali Aghagolzadeh, "Alzheimer's disease diagnosis from structural MRI using Siamese convolutional neural network", 2019 4th International Conference on Pattern Recognition and Image Analysis (IPRIA), 6 and 7 March, Tehran, Iran

12. Marie Chan, Bernard Andre, Armando Herrera, Pierre Celsis, "Incremental Learning in a Multilayer Neural Network as an Aid to Alzheimer's Disease Diagnosis", July 15, 1993.

13. Prof.Mrs.M.M.Patil, Prof.A.R.Yardi, "ANN based Dementia Diagnosis using DCT for Brain MR Image compression", International conference on Communication and Signal Processing, April 3-5, 2013, India.

14. M.Akin, M.A.Arserim, M.K.Kiymik, I.Turkoglu, “A NEW APPROACH FOR DIAGNOSING EPILEPSY BY USING WAVELET TRANSFORM AND NEURAL NETWORKS", 2001 Proceedings of the 23rd Annual EMBS International Conference October 25-28, Istanbul, Turkey. 


\section{Diagnosis of Brain Diseases using Neural Networks}

15. Khalil AlSharabi, Sutrisno Ibrahim, Ridha Djemal, Abdullah Alsuwailem, "A DWT-Entropy-ANN Based Architecture for Epilepsy Diagnosis Using EEG Signals", 2nd International Conference on Advanced Technologies for Signal and Image Processing - ATSIP 2016 March 21-24, 2016, Monastir, Tunisia

16. Mosarrat Rumman, Abu Nayeem Tasneem, Sadia Farzana, Monirul Islam Pavel, Dr. Md. Ashraful Alam, "Early detection of Parkinson's disease using image processing and artificial neural network", 2018 Joint 7th International Conference on Informatics, Electronics \& Vision (ICIEV) and 2018 2nd International Conference on Imaging, Vision \& Pattern Recognition (icIVPR).

17. Farhad Soleimanian Gharehchopogh, Peyman Mohammadi, "A Case Study of Parkinson's disease Diagnosis using Artificial Neural Networks", International Journal of Computer Applications (0975 8887), Volume 73- No.19, July 2013

\section{AUTHORS PROFILE}

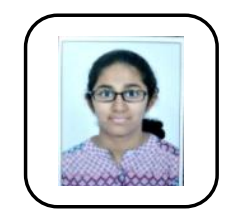

Ms. Anagha Naga Krishna, B.E. in Computer Science and Engineering at RNSIT, Bengaluru. Her research interests include image processing and pattern classification

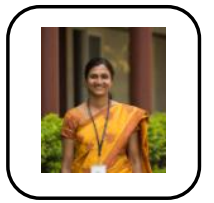

Dr. M J Sudhamani, working as an Assistant Professor in Dept. of Computer Science and Engineering at RNSIT, Bengaluru. Her research interests include image processing, biometric analysis. 